\title{
The Yeast Nucleosome Atlas (YNA) database: an integrative gene mining platform for studying chromatin structure and its regulation in yeast
}

\author{
Po-Cheng Hung ${ }^{1}$, Tzu-Hsien Yang ${ }^{1}$, Hung-Jiun Liaw ${ }^{2 *}$, Wei-Sheng $\mathrm{Wu}^{1^{*}}$ \\ From Asia Pacific Bioinformatics Network (APBioNet) Thirteenth International Conference on Bioinformatics \\ (InCoB2014) \\ Sydney, Australia. 31 July - 2 August 2014
}

\begin{abstract}
Background: Histone modification and remodeling play crucial roles in regulating gene transcription. These posttranslational modifications of histones function in a combinatorial fashion and can be recognized by specific histonebinding proteins, thus regulating gene transcription. Therefore, understanding the combinatorial patterns of the histone code is vital to understanding the associated biological processes. However, most of the datasets regarding histone modification and chromatin regulation are scattered across various studies, and no comprehensive search and query tool has yet been made available to retrieve genes bearing specific histone modification patterns and regulatory proteins.
\end{abstract}

Description: For this reason, we developed the Yeast Nucleosome Atlas database, or the YNA database, which integrates the available experimental data on nucleosome occupancy, histone modifications, the binding occupancy of regulatory proteins, and gene expression data, and provides the genome-wide gene miner to retrieve genes with a specific combination of these chromatin-related datasets. Moreover, the biological significance analyzer, which analyzes the enrichments of histone modifications, binding occupancy, transcription rate, and functionality of the retrieved genes, was constructed to help researchers to gain insight into the correlation among chromatin regulation and transcription.

Conclusions: Compared to previously established genome browsing databases, YNA provides a powerful gene mining and retrieval interface, and is an investigation tool that can assist users to generate testable hypotheses for studying chromatin regulation during transcription. YNA is available online at http://cosbi3.ee.ncku.edu.tw/yna/.

\section{Background}

The eukaryotic genome is packaged into nucleosomes, each of which consists of approximately 147 base pairs of DNA wrapped around a histone octamer and constitutes fundamental unit of the chromatin structure $[1,2]$. The histone octamer contains $\mathrm{H} 2 \mathrm{~A}, \mathrm{H} 2 \mathrm{~B}, \mathrm{H} 3$, and $\mathrm{H} 4$ proteins which are highly conserved and are subjected

\footnotetext{
* Correspondence: liawh@mail.ncku.edu.tw; wessonwu@gmail.com 'Department of Electrical Engineering, National Cheng Kung University, No.1 University Road, Tainan City 701, Taiwan

${ }^{2}$ Department of Life Sciences, National Cheng Kung University, No.1 University Road, Tainan City 701, Taiwan

Full list of author information is available at the end of the article
}

to post-translational modifications, including acetylation, methylation, phosphorylation, and ubiquitination [3-5]. Emerging evidence suggests that specifically modified histones function in a combinatorial patterns that can be read by corresponding domains of regulatory proteins and lead to specific cellular events, such as DNA replication and gene transcription [6-8]. In support of this notion, chromatin regulating complexes, such as histone modification enzymes and chromatin remodeling complexes, often contain multiple domains that can bind specifically modified histones. For example, the NuA4 complex, which is a histone acetyltransferase toward $\mathrm{H} 2 \mathrm{~A}$ and $\mathrm{H} 4$, plays an essential role in the transcription 
of ribosomal genes. Interestingly, it contains chromo, PHD, actin-related, and YEATS domains that interact with methylated H3K36, methylated H3K4, phosphorylated $\mathrm{H} 2 \mathrm{~A}$, and acetylated $\mathrm{H} 3$, respectively [9]. Another complex SWR1, which contains bromodomains interacting with acetylated $\mathrm{H} 3$ and $\mathrm{H} 4$, is a chromatin remodeling complex that catalyzes the exchange of histone H2A-H2B dimers with H2AZ-H2B dimers and plays an important role in genome integrity and DNA repair [10]. These multivalent interactions between domains and modified histones have led to the concept of the histone code hypothesis [11-13]. However, the question of how to decipher these combinatorial codes in relation to specific biological events has not been clearly answered. Therefore, there is an urgent need to combine all chromatin related datasets into one platform in order to facilitate biologists for in-depth analyses.

Previous studies have produce several valuable genome-wide datasets of histone modifications, binding occupancy of chromatin-regulating factors, and gene expression in the yeast Saccharomyces cerevisiae [14-21]. However, these datasets are scattering across the literatures and researchers are often suffering from searching these fragmentary datasets for further exploration. Despite that a number of databases have been established to perform genome-wide investigation for multiple biological features, there are still no suitable tool for gene mining based on the aspect of chromatin regulation. For example, Saccharomyces Genome Database (SGD) contains comprehensive biological information for Saccharomyces cerevisiae [22,23]. SGD also contains the genome browser, GBrowse [24], to display diverse experimental results, including chromatin-regulating features, and to achieve comprehensive genomic overview. Users can view specific regulation of chromatin structure in the specified regions by GBrowse. However, users cannot seek specific gene groups with specifically modified histones or specifically bound factors. Furthermore, it is difficult to correlate the regulations of chromatin structure with transcriptional expression and its binding factors. These information can be extracted only by analyzing the whole genome data through complicated computational procedures. Beside SGD, YeastMine, constructed collaboratively by SGD and the Intermine, is a data search and retrieval tool that incorporates various types of data present in SGD and provides custom query capabilities related to chromosomal features, sequences, protein features, GO annotations, phenotypes, interaction data, expression data, and curated literature [25]. Yet the query engines in YeastMine mainly focus on individual genes and their interactive relationship. Researchers concentrating on chromatin structure cannot find groups of genes sharing the same chromatin regulation pattern from YeastMine directly. Another database, ChromatinDB, provides the query engine to facilitate statistical analysis and visualization of chromatin features based on user-specified gene sets or specific chromosomal regions [26]. The chromatin visualization function on ChromatinDB displays the enrichment of deposited histone modification patterns graphically and further indicates the potential covalent modifications on histones for a given gene sets. However, users need to provide pre-selected gene lists for the analysis. Furthermore, ChromatinDB cannot provide the gene mining function to fetch genes with similar histone modification patterns and associated factors.

Hence we developed the Yeast Nucleosome Atlas database, or the YNA database, to integrate available chromatin related datasets into one platform and to provide a comprehensive query and investigation tool. In YNA, approximately 100 deposited data tracks of nucleosome occupancy, histone modifications, binding occupancy of chromatin-related regulatory proteins are integrated in the database. Besides, YNA also collected global gene expression data and MIPS FunCat functional catalogue for functional analysis. Most importantly, YNA provides the genome-wide gene miner to retrieve genes with user-specified filtering criteria based on the collected datasets. The retrieved genes can be utilized for further analysis. Furthermore, YNA also provides the biological significance analyzer to present the tendency of these chromatin-regulating features for the retrieved genes. This helps users obtain hypotheses to investigate chromatin structure and transcription regulation. Therefore, YNA is a discovery tool that provides the comprehensive investigation for chromatin structure, and can help users propose testable hypotheses for studying chromatin regulation during transcription. YNA is available online at http://cosbi3.ee.ncku.edu.tw/yna/.

\section{Construction and contents}

The construction and architecture of YNA are shown in Figure 1. YNA is constructed based on the datasets of chromatin structure regulations, such as histone modifications, histone variants and regulatory protein binding events. These data are reprocessed into genomic sequences of individual genes and eliminated the noncoding sequences. Other datasets like gene expression data and MIPS FunCat functional categories are arranged with the summary information of individual gene. These collected datasets are described in the subsection "Data collection and processing". To acquire the gene query and search interface, the genome-wide gene miner was implemented. We also developed the biological significance analyzer for further analysis of the retrieved genes. These two features are described in the subsection of "Genome-wide gene miner" and "Biological significance analyzer", respectively. 


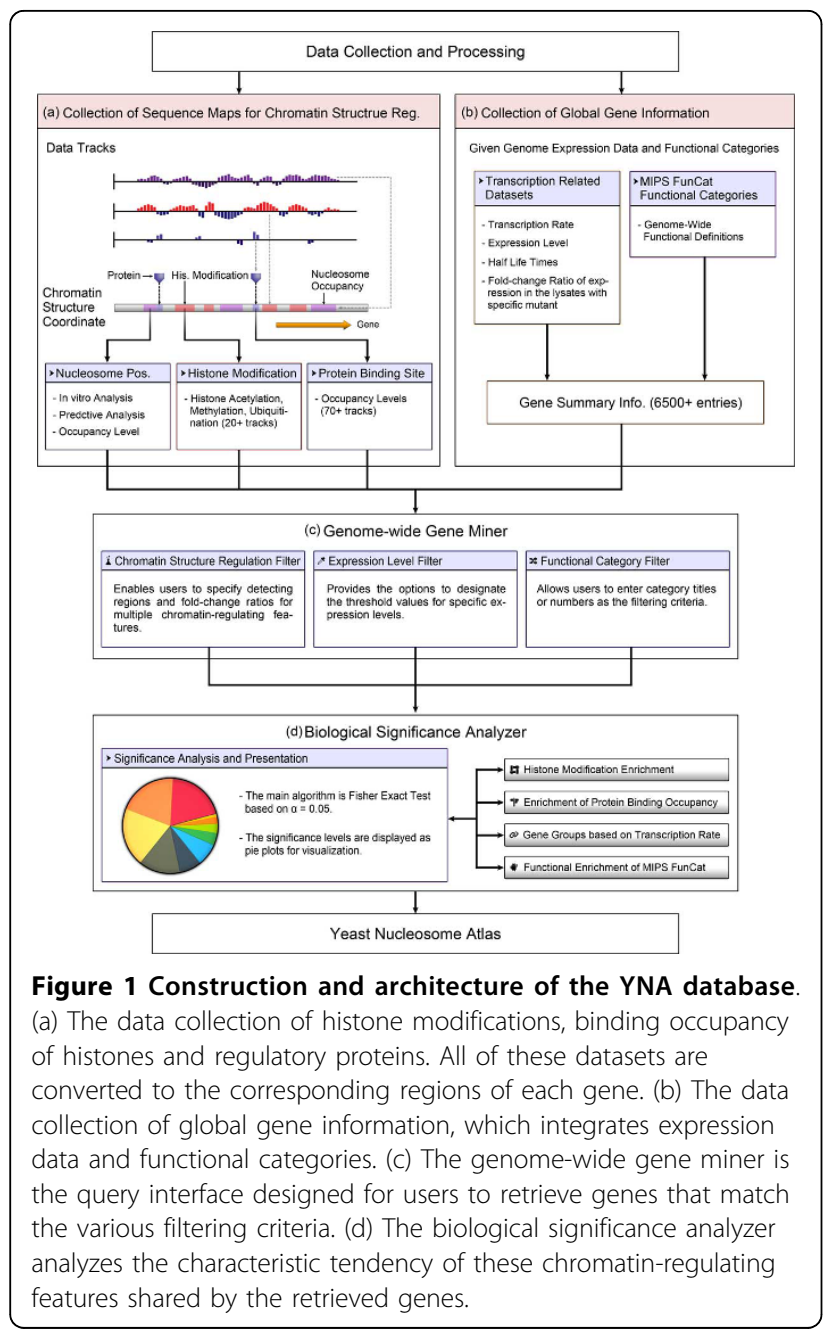

\section{Data collection and processing}

In YNA, all collected datasets can be classified into 8 classes based on the properties. These 8 classes are the genome summary information, the nucleosome positioning data, the histone modification data, the histone variant data, the binding occupancy data of regulatory proteins, the gene expression data, the expression data of mutant cells, and the data of MIPS functional category.

\section{Yeast gene collections}

The yeast genome summary was retrieved from Saccharomyces Genome Database (SGD) [22,23]. The gene summary includes the systematic name, gene alias, gene description and coordinates of coding regions for about 6500 genes.

\section{Nucleosome positioning data}

Three datasets of genome-wide nucleosome organization maps are included in the YNA database [14-16]. In YNA, the datasets of nucleosome organization are displayed as sequence coordinate charts with corresponding predicted occupancy scores or normalized detection values of nucleosome occupancy.

\section{Histone modification data}

Post-translational modifications of histones play crucial roles in altering chromatin structure and creating marks for the binding sites of specific chromatin regulatory proteins, thereby regulating genomic expression behaviors [5]. In YNA, approximately 20 different data tracks related to histone modification were collected. These data present the modification states in the formats of enrichment levels for the whole genome. The main composition of histone modification patterns employed in YNA are histone acetylation (acetyl-lysine 4 of histone H3 (H3K4ac), acetyllysine 9 of histone H3 (H3K9ac), acetyl-lysine 14 of histone $\mathrm{H} 3$ (H3K14ac), and histone $\mathrm{H} 4$ acetylation (H4ac)), methylation (monomethyl-lysine 4 of histone $\mathrm{H} 3$ (H3K4me), dimethyl-lysine 4 of histone H3 (H3K4me2), trimethyl-lysine 4 of histone H3 (H3K4me3), trimethyllysine 36 of histone $\mathrm{H} 3$ (H3K36me3), dimethyl-lysine 79 of histone $\mathrm{H3}$ (H3K79me2), and trimethyl-lysine 79 of histone $\mathrm{H} 3$ (H3K79me3)) and ubiquitination (monoubiquitination of histone H2B on lysine 123 (H2BK123ub)) [17-20]. In addition, data tracks of histone modifications in mutant cells, such as set $1 \Delta, u b p 8 \Delta$, and $u b p 10 \Delta$, are also included.

\section{Data of binding occupancy of regulatory proteins}

In eukaryotes, transcription is regulated by hundreds of proteins. These proteins consist of sequence-specific DNA-binding proteins, chromatin regulators, elongation regulators and transcription factors $[27,28]$. We gathered the data of binding occupancy of 73 proteins, which belong to proteins of chromatin remodeling and histone modifications [21]. In YNA, the data of binding occupancy of regulatory proteins are displayed as the coordinate charts with fold-change ratios over the backgrounds. The fold-change ratios indicate the binding enrichments of regulatory proteins in the selected regions.

\section{H2A.Z histone variant data}

H2A.Z, the histone variant of $\mathrm{H} 2 \mathrm{~A}$, has been widely studied and is thought to regulate several biological processes. The global H2A.Z map is also included in the YNA database [29].

\section{Gene expression data}

The expression data of each gene are collected in YNA [30]. The adopted dataset has three types of expression data under YPD growth condition. The three expression data are the transcription rate of mRNA per hour, the expression level calculated from mRNA copies per cell, and the half life time of mRNA, respectively.

\section{Data of expression profiles of mutant cells}

The expression data of mutant cells, including arp $8 \Delta$, ino80A, set1A, and H3K4R (the replacement of lysine 4 with arginine in histone $\mathrm{H} 3$ ), are also collected in YNA $[17,31]$. Ino80 and Arp8 are two subunits of INO80 
complex, which is an ATP-dependent chromatin remodeler. Mutations in the ino80 $\Delta$ and arp $8 \Delta$ genes are defective in DNA double-strand break repair and in gene transcription. The dataset includes the fold-change of expression levels in the ino80 $\Delta$ and arp $8 \Delta$ mutant cells, compared to wild type cells.

\section{Data of MIPS FunCat functional annotation}

MIPS FunCat (MIPS Functional Catalogue) [32,33] is a functional annotation scheme with systematic classification and clear definitions for the gene functions. FunCat consists of 28 main categories, which are hierarchically structured and contain multiple child nodes representing distinct functions in several levels. In YNA, we retained about 500 FunCat catalogue nodes with entries matching in yeast genes and integrated them into the gene summary information.

\section{Genome-wide gene miner}

We implemented the genome-wide gene miner in YNA to retrieve genes that match the filtering criteria. The genome-wide miner has three main filter set, which are chromatin structure regulation filter, expression level filter, and functional category filter, respectively. The three filters can be used with combination.

\section{Chromatin structure regulation filter}

The main purpose of chromatin structure regulation filter is to query for genes that match specific chromatin-regulating features, such as histone modifications, histone variants and binding occupancy of regulatory proteins, in promoters or coding regions. In this filter set, users are able to specify the threshold ratios relative to the background. The region options consist of (I) coding region of genes, (II) promoter region, which is defined between 500 bp upstream and $100 \mathrm{bp}$ downstream from the gene start site, (III) both coding and promoter regions, and (IV) either of the two regions, separately. To achieve better query performance, we reprocessed all data from original sequence-formatted rows into individual gene regions and eliminated those non-coding sequences to abandon superfluous noises. While executing the query process, YNA globally traverses all sample values inside targeted regions from the rearranged data to filter out those target genes matching the defined criteria.

\section{Expression level filter}

The format of expression data deposited in YNA are measured by each gene and can be roughly divided into two types. The first type is quantified transcriptional property such as transcription rate and the counts of mRNA copies per cell, which directly deliver the information of gene's capability [30]. The other one is the difference ratio of expression level under specific induced mutants (ino80 4 , $\operatorname{arp} 8 \Delta$, set1, H3K4R) comparing to wild types $[17,31]$. Expression level filter operates with these datasets based on numeric filtering, where users can define the threshold values and mathematical operators for each expression feature as the filtering criterion in this filter set.

\section{Functional category filter}

The functional category filter provides the interface to fetch those genes that match the MIPS Functional Catalogue. In this filter set, users are able to specify keywords or category numbers to retrieve those genes with matching functional categories.

\section{Biological significance analyzer}

The genome-wide gene miner provides the interface for users to extract genes that match the query criteria. To gain insight into the correlation between chromatin structure and transcription regulation of the retrieved genes, we implemented the biological significance analyzer along with the query results. The analyzer provides the enrichment analyses in four aspects: (I) the enrichment of histone modifications, which calculates the significance levels of the specific histone modification patterns in promoters and coding regions; (II) the enrichment of binding occupancy of regulatory proteins, which calculates the significance levels of regulatory protein binding events in promoters and coding regions; (III) mRNA transcription rate, which evaluates the significance levels of the transcription rate of the retrieved genes. The transcription rate is divided into 5 groups: "greater than $50 \mathrm{mRNA} / \mathrm{hr}$ ", "16 - $50 \mathrm{mRNA} / \mathrm{hr}$ ", "4 $16 \mathrm{mRNA} / \mathrm{hr}$ ", "1 - $4 \mathrm{mRNA} / \mathrm{hr}$ ", and "less than 1 mRNA/hr"; and (IV) the functional enrichment of MIPS functional categories of the retrieved genes.

The main algorithm to analyze the significance is based on Fisher Exact Test. Fisher Exact Test is used for analyzing the statistical significance of the proportion representing some categorized data subsets, such as those classified feature patterns mentioned above, within a given gene list relative to the genomic abundance ratio with the same property [34]. YNA adopts a statistic confidence level of $\alpha$ $=0.05$. To acquire more visual conception, the calculated $p$-values in the four analyses are converted into positive correlated scores by taking the negative logarithm and are then displayed as pie plots.

The biological significance analyzer only displays those significantly enriched features (we adopted a two times fold-change ratio as the enrichment threshold for all features). The resulting analysis demonstrates the tendency of these features shared by the retrieved genes. Moreover, this provides hypotheses for the combinatorial effects of those significant chromatin-regulating features for downstream analysis.

\section{Utility and discussions \\ Database implementation}

The construction of YNA is based on CodeIgniter PHP Framework and MySQL database management system. 
The design layout of website interface was extended from YAML CSS Framework and jQWidgets. To improve the browse experience and performance, we developed vast JavaScript (ECMAScript) applications. Furthermore, all query operations in YNA are achieved in REST (Representational state transfer) architectural style. The sequence maps of chromatin structure regulations are presented as static images, which are produced by MatplotLib, a library of the Python scripting language.

\section{Database interface}

The YNA database provides three main functional modes for users to investigate the regulations for chromatin structure: (I) The query mode contains the manipulation panel of the genome-wide gene miner and the gene query engine (Figure 2a); (II) The list mode presents the list of retrieved genes that match the query criteria (Figure 2b). The biological significance analyzer displays the analysis results of the retrieved genes as pie plots (Figure 2c); (III) The detail mode exhibits the basic description and the chromatin-related patterns of the specified gene (Figure 2d). The query mode is set up in the query page, and the other two modes are integrated in the result page. A functional panoramic view of the YNA database is shown in Figure 2.

The genome-wide gene miner is the main function in the query mode. Users are able to specify filtering criteria based on the three filter sets, i.e. chromatin structure regulation filter, expression level filter, and functional category filter, and then execute the query to retrieve genes that match the filtering criteria (Figure $2 a)$. The chromatin structure regulation filter enables users to specify coding or promoter regions and foldchange ratios of specific histone modifications and binding occupancy of regulatory proteins. The expression level filter provides the options for users to specify threshold values of expression levels. The functional category filter allows users to enter MIPS FunCat category keywords or numbers to search genes that match the search criteria. YNA also provides query defaults, which are pre-defined filter settings, so that users can easily get started. Furthermore, we also constructed the traditional gene query engine in the query mode, which enables users to search individual genes by their systematic names or standard names.

In the list mode, the retrieved genes are displayed in the table. The genes are also analyzed through the biological significance analyzer in terms of four aspects: histone modifications, binding occupancy of regulatory proteins, transcription rates, and functional categories. All of the displayed table contents and charts can be downloaded in multiple file types (Excel table, CSV table, XML format and JSON format). The interface of the list table and biological significance analyzer (a)

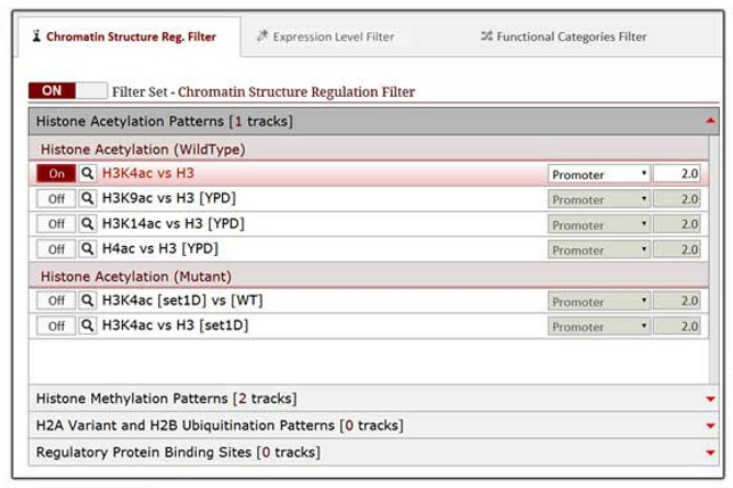

(c)

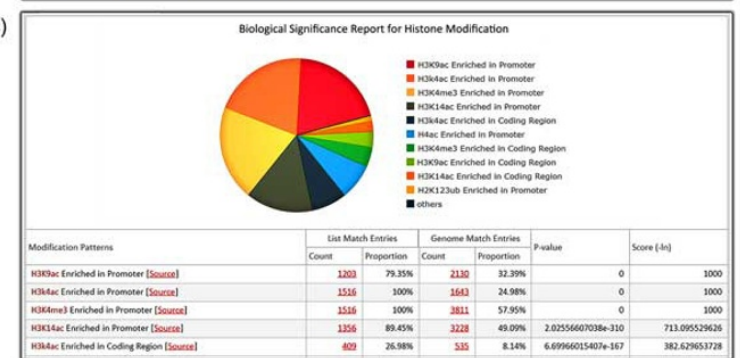

(b)

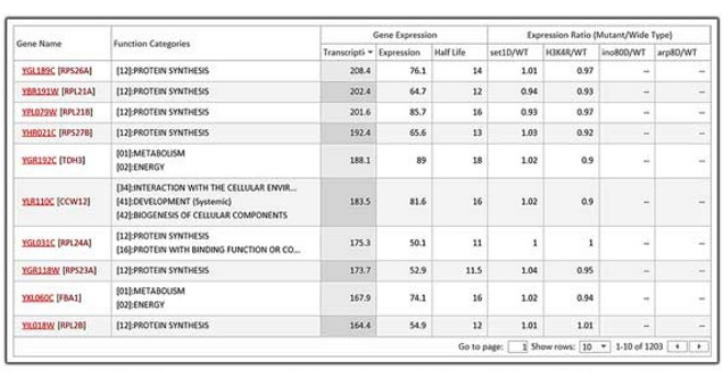

(d)

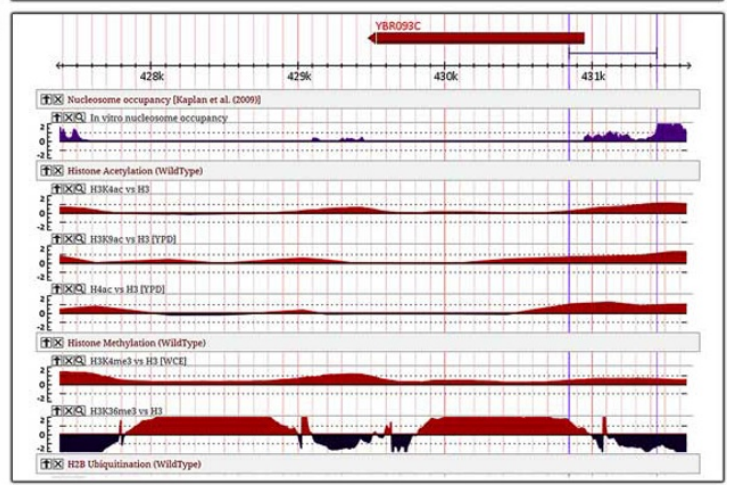

Figure 2 The functional panoramic view of YNA database. (a) The snapshots of genome-wide gene miner. (b) The gene list is retrieved through the genome-wide gene miner. The table information includes the gene name, functional categories, expression levels, and expression differences between wild type and mutant cells. (c) The biological significance analyzer provides the significance analysis of the retrieved genes and displays the results as pie plots. (d) The sequence maps of histone modifications in the specified gene region. 
mounted in list mode can be viewed in Figures 2b and Figure 2c.

In the detail mode, the summary of the specifically selected gene is exhibited. The summary includes the information of the specified gene and sequence maps of multiple chromatin-related datasets, including nucleosome positioning, histone modifications and the binding occupancy of regulatory proteins, near the gene region. These sequence maps are shown in $\log 2$ fold-change ratios to represent the enrichment or depletion of specific chromatin regulation patterns (Figure 2d).

All processed plain text files of chromatin-related datasets are annotated with their sources and are available for downloading in the statistics page. We also provided a detailed tutorial page so that users can fully utilize functions in YNA.

\section{Case study}

To demonstrate the usability of YNA, we query genes with specific filtering criteria in the genome-wide gene miner. The retrieved genes are further analyzed in the biological significance analyzer to gain insight into information of the retrieved genes. In S. cerevisiae, histone acetylation and methylation are widely studied and are thought to regulate gene transcription [8]. Hence we took following two examples describing the functionality of YNA.

\section{Acetyl-lysine 4 of histone $\mathrm{H3}$ (H3K4ac) and trimethyl-lysine 4 of histone $\mathrm{H3}$ (H3K4me3)}

Histone modifications, such as the modifications of $\mathrm{H} 3 \mathrm{~K} 4 \mathrm{ac}$ and H3K4me3 enriched in promoters, have long been known to be associated with actively transcribed genes. Therefore, we set the chromatin structure regulation filter of the genome-wide gene miner, such that both H3K4ac and H3K4me3 must have fold-changes equal to or greater than 2 folds in promoters. 1516 matched genes were retrieved (Figure 3a). Consistent with previously published results, we discovered that these genes are transcribed either at a high level (greater than $50 \mathrm{mRNA} / \mathrm{hr}$ ) or at a mid-high level (16 - $50 \mathrm{mRNA} / \mathrm{hr})$, with $p$-values of $10^{-93}$ and $10^{-28}$, respectively, through the biological significance analyzer (Figure $3 \mathrm{~b}$ ) $[17,20]$. In addition, the acetyl-K9 of histone $\mathrm{H} 3$ (H3K9ac), the acetyl-K14 of histone H3 (H3K14ac), and $\mathrm{H} 4$ acetylation are also enriched in promoters of the 1516 genes (Figure 3c). Consistent with previously published results, H3K9ac, H3K14ac, and H4ac were also shown to be associated with highly transcribed genes $[20,27,35]$.

Furthermore, we discovered that several regulatory proteins, including Swi3, Rsc1, and Ino80, are associated with promoters of these genes with $p$-values of $10^{-56}$, $10^{-43}$, and $10^{-42}$, respectively (Figure $3 \mathrm{~d}$ ) [36]. These regulatory proteins were known to be involved in chromatin remodeling. Finally, applying functional category analysis to the 1516 genes, most were found to participate in ribosomal protein synthesis and metabolism related enzyme synthesis, which are necessary for the translational machinery (Figure 3e) [8].

\section{Histone $\mathrm{H} 4$ acetylation (H4ac)}

Histone $\mathrm{H} 4$ acetylation enriched in promoters, has long been considered to associate with transcription. Therefore, we set the filtering criterion in the genome-wide gene miner that $\mathrm{H} 4 \mathrm{ac}$ has fold-changes equal or greater

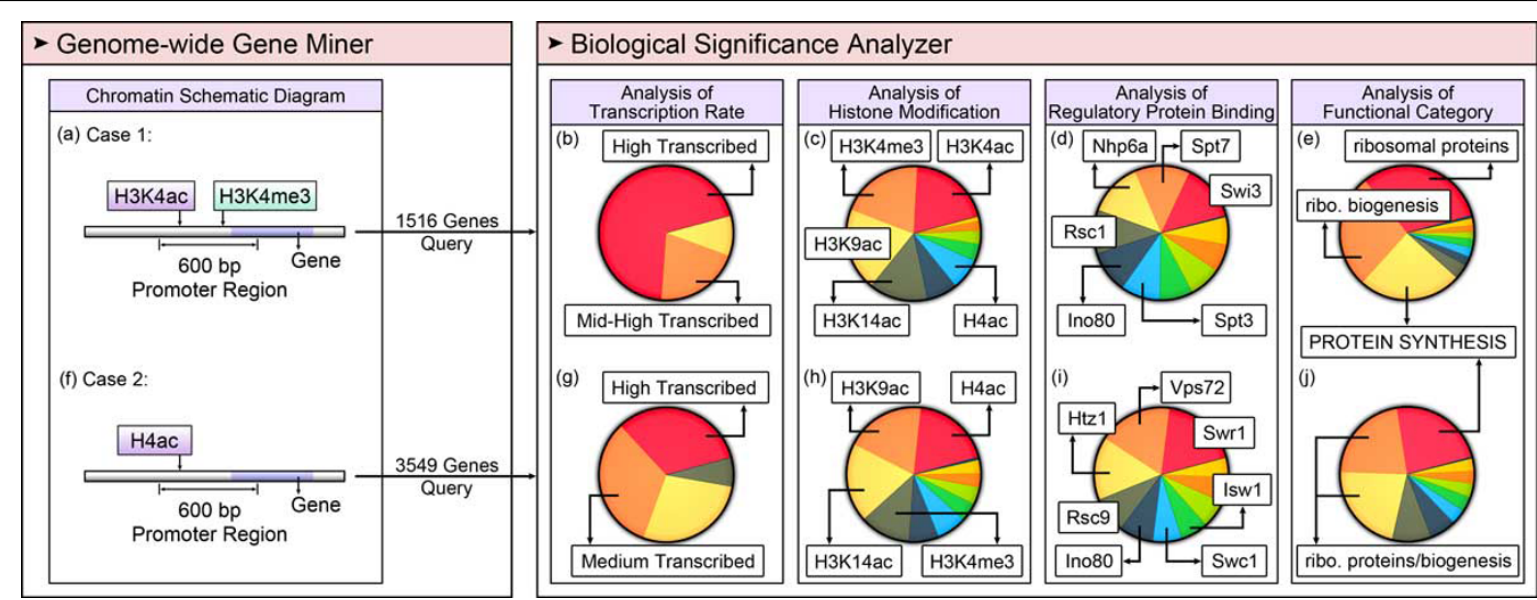

Figure 3 Case studies using the YNA database. (a) A combined search of H3K4aC and H3K4me3 in the promoter region in the genome-wide gene miner of the YNA, which leads to the retrieval of 1516 genes that match the query criteria. The retrieved genes were further analyzed using the biological significance analyzer, which displays the enrichments of the histone modifications (b), the enrichments of the regulatory protein binding occupancy (c), the transcription rate in 5 groups (d), and the enrichments of the functional categories (e) of the retrieved genes. (f) Another search of $\mathrm{H} 4 \mathrm{ac}$ enriched in the promoter region, which results in the retrieval of 3549 genes that match the query criteria. ( $\mathrm{g}-\mathrm{j}$ ) The biological significance analyzer analyzes these retrieved genes and provides the enrichment analyses in the four aspects. 
than 2 fold in promoters, and we retrieved 3549 matching genes after the query (Figure $3 \mathrm{f}$ ). In the biological significance analyzer, we discovered that these genes are transcribed either at high level (greater than 50 mRNA/ $\mathrm{hr}$ ) or at medium level (4-16 mRNA/hr) with $p$-values of $10^{-18}$, and this result is consistent with previously published results (Figure 3g) [5]. Besides, some modification patterns, such as H3K9ac, H3K14ac, and H3K4me3, are also enriched in promoters of these genes with $p$-value less than $10^{-277}$ (Figure $3 \mathrm{~h}$ ). This suggested the potential that H3K9ac, H3K14ac, and H3K4me3 are also associated with highly transcribed genes, which are also supported by previous studies [20,27].

Furthermore, several regulatory proteins, including Swr1, Vps72, Rvb1, and Esa1, were found to be associated with promoters of these genes with $p$-value less than $10^{-12}$ (Figure 3i). Swr1, Vps72, and Rvb1 belong to SWR1 complex, whereas Esa1 belongs to NuA4 complex. NuA4 is a histone acetyltransferase toward H2A and H4. Therefore, the enrichment of Esa1 is consistent with the enrichment of H4ac [8]. Interestingly, NuA4 complex is highly conserved from yeast to human. TIP60 is human homolog of yeast NuA4 complex. TIP60 corresponds to a near-exact merge of yeast NuA4 and SWR1 complexes $[37,38]$. The finding that Swr1, Vps72, and Rvb1 are also enriched in promoter as the enrichment of H4ac, suggesting that NuA4 and SWR complexes might work together to regulate transcription in yeast [39].

\section{Summary}

The two case studies demonstrated that histone acetylation (H3K4ac and H4ac) and methylation (H3K4me3) are highly correlated with transcription activities [8]. Moreover, the biological significance analyzer provides testable hypotheses, which have been validated in the literatures. Thus, YNA provides a platform for researchers to investigate chromatin regulations, and to generate testable hypotheses that can be further verified in experiments.

\section{Issues related to YNA}

YNA deposits genome-wide chromatic-regulation datasets for elucidating the transcriptional mechanisms caused by alterations on chromatin structure. However, these datasets came from several studies, and the experimental procedures may vary from one laboratory to another, thus resulting in potential systematic biases. In systems biology, a cellular system is perturbed and measured by high-throughput technologies [40]. Hence, the integration of different high-throughput assays, which were probably performed on different cell states, suffers from the systematic bias and unpredictable noises $[41,42]$. Since this type of systematic bias is still unavoidable [41], some crucial points are worthy of attention when browsing these regulating identification of chromatin structure.

In YNA, we collected the datasets of histone modifications, protein binding occupancy and expression data from multiple studies. In these data, the yeast strains used for chromatin immunoprecipitation or expression profiling experiments are derived either from yeast $\mathrm{Sac}$ charomyces cerevisiae strain S288c or W303, which share very similar genetic background and culture condition (cells growing to $\log$-phase at $30^{\circ} \mathrm{C}$ in YPD). Hence the systematic bias of condition variation in the data integration of YNA is not severe. Besides, to provide more detailed information for each dataset, we have marked out the experimental environments and the assay resolutions for which the high-throughput experiments were performed.

On the other hand, it is well known that post-translational modifications of histones, particularly acetylation and methylation, play wide-spread roles in transcription regulations. But nowadays the genome-wide sequence maps of histone modification are still not completely available for all possible combinations of those features in all different cellular conditions [5,8]. Furthermore, studies focusing on histone modifications so far have examined modifications in cells that are cycling asynchronously and are growing in rich media. Therefore, these datasets are simply a snapshot of histone modifications at one point during transcription [5]. This fact limits the possibility that histone modification status might change in response to environmental stresses. In the current version of YNA, we aimed to develop the visual and analysis tool for genome-wide chromatin structure regulations. And all of these analyses are based on steady-state with respect to gene expression. We will incorporate more comprehensive data collection as the updating plan when new datasets related to histone modifications in different cellular conditions are published in the future.

\section{Availability and requirements}

YNA is available at http://cosbi3.ee.ncku.edu.tw/yna/. We recommend users to browse YNA by Google Chrome. Other modern browsers such as Microsoft IE9, Apple Safari and Mozilla Firefox 21 also perform well in YNA. JavaScript is required in YNA functionality and should be enabled.

\section{Conclusions}

In this study, we developed the YNA database, which integrates a number of scattered datasets of histone modifications, binding occupancy of regulatory proteins, gene expression data, and functional categories. Most importantly, YNA provides the genome-wide gene miner that allows user to retrieve genes based on 
combinatorial filtering criteria of specific histone modification patterns, expression levels, and functional categories. These retrieved genes can be further analyzed using the biological significance analyzer for the enrichments of specific chromatin-related features. Therefore, YNA is an investigation and discovery tool that can be used to mine proper targeted genes and to propose testable hypotheses for further chromatin studies. YNA will be updated regularly to keep with the latest chromatin-regulation datasets.

\section{List of abbreviations}

SGD, Saccharomyces Genome Database; MIPS, Munich Information Center for Protein Sequence; FunCat, Functional Catalogue; YNA, Yeast Nucleosome Atlas database.

\section{Competing interests}

The authors declare that they have no competing interests.

\section{Authors' contributions}

$\mathrm{PCH}$ processed collected datasets and constructed YNA website. PCH and THY wrote the manuscript. HJL provided essential guidance and inspected biological issues. HJL and WSW conceived the research topics. PCH, THY and HJL proofread the manuscript. All authors have read and approved the final manuscript.

\section{Acknowledgements}

This study is funded by grants (NSC102-2320-B-006-037, NSC101-2320-B-006039, NSC100-2320-B-006-016, NSC100-2311-B-006-001 and MOST-103-2221-E006-174-MY2) from the Ministry of Science and Technology, the Ministry of Education, Taiwan, and the Aim for the Top University Project to the National Cheng Kung University.

\section{Declarations}

The publication of this supplement was funded by the Aim for the Top University Project to the National Cheng Kung University.

This article has been published as part of BMC Genomics Volume 15 Supplement 9, 2014: Thirteenth International Conference on Bioinformatics (InCoB2014): Computational Biology. The full contents of the supplement are available online at http://www.biomedcentral.com/bmcgenomics/ supplements/15/59.

\section{Authors' details}

'Department of Electrical Engineering, National Cheng Kung University, No.1 University Road, Tainan City 701, Taiwan. ${ }^{2}$ Department of Life Sciences, National Cheng Kung University, No.1 University Road, Tainan City 701, Taiwan.

\section{Published: 8 December 2014}

\section{References}

1. Noll M, Kornberg RD: Action of micrococcal nuclease on chromatin and the location of histone H1. Journal of Molecular Biology 1977, 109(3):393-404.

2. Richmond TJ, Davey CA: The structure of DNA in the nucleosome core. Nature 2003, 423(6936):145-150.

3. Cheung WL, Briggs SD, Allis CD: Acetylation and chromosomal functions. Current Opinion in Cell Biology 2000, 12(3):326-333.

4. Martin C, Zhang Y: The diverse functions of histone lysine methylation. Nature Reviews Molecular Cell Biology 2005, 6(11):838-849.

5. Millar $\mathrm{CB}$, Grunstein M: Genome-wide patterns of histone modifications in yeast. Nature Reviews Molecular Cell Biology 2006, 7(9):657-666.

6. Kornberg RD, Lorch Y: Twenty-five years of the nucleosome, fundamental particle of the eukaryote chromosome. Cell 1999, 98(3):285-294.

7. Boeger H, Griesenbeck J, Kornberg RD: Nucleosome retention and the stochastic nature of promoter chromatin remodeling for transcription. Cell 2008, 133(4):716-726.
8. Rando OJ, Winston F: Chromatin and transcription in yeast. Genetics 2012, 190(2):351-387.

9. Ginsburg DS, Govind CK, Hinnebusch AG: NuA4 lysine acetyltransferase Esa1 is targeted to coding regions and stimulates transcription elongation with Gcn5. Molecular and Cellular Biology 2009, 29(24):6473-6487.

10. Mizuguchi G, Shen X, Landry J, Wu WH, Sen S, Wu C: ATP-driven exchange of histone H2AZ variant catalyzed by SWR1 chromatin remodeling complex. Science 2004, 303(5656):343-348.

11. Liu CL, Kaplan T, Kim M, Buratowski S, Schreiber SL, Friedman N, Rando OJ: Single-nucleosome mapping of histone modifications in S. cerevisiae. PLoS Biology 2005, 3(10):e328.

12. Kouzarides T: Chromatin modifications and their function. Cell 2007, 128(4):693-705.

13. Rando OJ: Combinatorial complexity in chromatin structure and function: revisiting the histone code. Current Opinion in Genetics and Development 2012, 22(2):148-155.

14. Kaplan N, Moore IK, Fondufe-Mittendorf Y, Gossett AJ, Tillo D, Field Y, LeProust EM, Hughes TR, Lieb JD, Widom J, et al: The DNA-encoded nucleosome organization of a eukaryotic genome. Nature 2009, 458(7236):362-366

15. Field $Y$, Kaplan N, Fondufe-Mittendorf $Y$, Moore IK, Sharon E, Lubling $Y$, Widom J, Segal E: Distinct modes of regulation by chromatin encoded through nucleosome positioning signals. PLoS Computational Biology 2008, 4(11):e1000216.

16. Lee W, Tillo D, Bray N, Morse RH, Davis RW, Hughes TR, Nislow C: A highresolution atlas of nucleosome occupancy in yeast. Nature Genetics 2007, 39(10):1235-1244.

17. Guillemette $B$, Drogaris $P$, Lin $H H S$, Armstrong $H$, Hiragami-Hamada $K$, Imhof A, Bonneil E, Thibault P, Verreault A, Festenstein RJ: H3 lysine 4 is acetylated at active gene promoters and is regulated by $\mathrm{H} 3$ lysine 4 methylation. PLoS Genetics 2011, 7(3):e1001354.

18. Schulze JM, Hentrich T, Nakanishi S, Gupta A, Emberly E, Shilatifard A, Kobor MS: Splitting the task: Ubp8 and Ubp10 deubiquitinate different cellular pools of H2BK123. Genes and Development 2011, 25(21):2242-2247.

19. Kirmizis A, Santos-Rosa H, Penkett CJ, Singer MA, Vermeulen M, Mann M Bähler J, Green RD, Kouzarides T: Arginine methylation at histone H3R2 controls deposition of H3K4 trimethylation. Nature 2007, 449(7164):928-932.

20. Pokholok DK, Harbison CT, Levine S, Cole M, Hannett NM, Lee TI, Bell GW, Walker K, Rolfe PA, Herbolsheimer E, et al: Genome-wide map of nucleosome acetylation and methylation in yeast. Cell 2005, 122(4):517-527.

21. Venters $B$, Wachi $S$, Mavrich $T$, Andersen B, Jena $P$, Sinnamon A, Jain $P$, Rolleri N, Jiang C, Hemeryck-Walsh C, Pugh B: A comprehensive genomic binding map of gene and chromatin regulatory proteins in Saccharomyces. Molecular Cell 2011, 41(4):480-492.

22. Cherry JM, Adler C, Ball C, Chervitz SA, Dwight SS, Hester ET, Jia Y, Juvik G, Roe T, Schroeder M, et al: SGD: Saccharomyces genome database. Nucleic Acids Research 1998, 26:73-79.

23. Cherry JM, Hong EL, Amundsen C, Balakrishnan R, Binkley G, Chan ET, Christie KR, Costanzo MC, Dwight SS, Engel SR, et al: Saccharomyces Genome Database: the genomics resource of budding yeast. Nucleic Acids Research 2012, 40(D1):D700-D705.

24. Chan ET, Cherry JM: Considerations for creating and annotating the budding yeast Genome Map at SGD: a progress report. Database: the journal of biological databases and curation 2012.

25. Balakrishnan R, Park J, Karra K, Hitz BC, Binkley G, Hong EL, Sullivan J, Micklem G, Cherry JM: YeastMine - an integrated data warehouse for Saccharomyces cerevisiae data as a multipurpose tool-kit. Database: the journal of biological databases and curation 2012.

26. O'Connor TR, Wyrick JJ: ChromatinDB: a database of genome-wide histone modification patterns for Saccharomyces cerevisiae. Bioinformatics 2007, 23(14):1828-1830.

27. Li B, Carey M, Workman JL: The role of chromatin during transcription. Cell 2007, 128(4):707-719.

28. Venters BJ, Pugh BF: How eukaryotic genes are transcribed. Critical Reviews in Biochemistry and Molecular Biology 2009, 44(2-3):117-141.

29. Guillemette B, Bataille AR, Gévry N, Adam M, Blanchette M, Robert F, Gaudreau L: Variant histone H2A. Z is globally localized to the promoters of inactive yeast genes and regulates nucleosome positioning. PLOS Biology 2005, 3(12):e384. 
30. Holstege F, Jennings E, Wyrick J, Lee T, Hengartner C, Green M, Golub T, Lander E, Young R: Dissecting the regulatory circuitry of a eukaryotic genome. Cell 1998, 95:717-728.

31. van Attikum H, Fritsch O, Hohn B, Gasser SM: Recruitment of the INO80 complex by H2A phosphorylation links ATP-dependent chromatin remodeling with DNA double-strand break repair. Cell 2004, 119(6):777-788.

32. Mewes HW, Frishman D, Güldener U, Mannhaupt G, Mayer K, Mokrejs M, Morgenstern B, Münsterkötter M, Rudd S, Weil B: MIPS: a database for genomes and protein sequences. Nucleic Acids Research 2002, 30:31-34.

33. Ruepp A, Zollner A, Maier D, Albermann K, Hani J, Mokrejs M, Tetko I, Güldener U, Mannhaupt G, Münsterkötter M, et al: The FunCat, a functional annotation scheme for systematic classification of proteins from whole genomes. Nucleic Acids Research 2004, 32(18):5539-5545.

34. Upton GJ: Fisher's exact test. Journal of the Royal Statistical Society Series A Statistics in Society 1992, 155(3):395-402.

35. Shogren-Knaak M, Ishii H, Sun JM, Pazin MJ, Davie JR, Peterson CL: Histone H4-K16 acetylation controls chromatin structure and protein interactions. Science 2006, 311(5762):844-847.

36. Fazzio TG, Tsukiyama T: Chromatin remodeling in vivo: evidence for a nucleosome sliding mechanism. Molecular Cell 2003, 12(5):1333-1340.

37. Doyon Y, Côté J: The highly conserved and multifunctional NuA4 HAT complex. Current Opinion in Genetics and Development 2004, 14(2):147-154.

38. Doyon Y, Selleck W, Lane WS, Tan S, Côté J: Structural and functional conservation of the NuA4 histone acetyltransferase complex from yeast to humans. Molecular and Cellular Biology 2004, 24(5):1884-1896.

39. Krogan NJ, Baetz K, Keogh MC, Datta N, Sawa C, Kwok TC, Thompson NJ, Davey MG, Pootoolal J, Hughes TR, et al: Regulation of chromosome stability by the histone $\mathrm{H} 2 \mathrm{~A}$ variant $\mathrm{Htz} 1$, the Swr1 chromatin remodeling complex, and the histone acetyltransferase NuA4. Proceedings of the National Academy of Sciences of the United States of America 2004, 101(37):13513-13518.

40. Ideker T, Galitski T, Hood L: A new approach to decoding life: systems biology. Annual Review of Biochemistry 2001, 2:343-372.

41. Hwang D, Rust AG, Ramsey S, Smith JJ, Leslie DM, Weston AD, De Atauri P, Aitchison JD, Hood L, Siegel AF, et al: A data integration methodology for systems biology. Proceedings of the National Academy of Sciences of the United States of America 2005, 102(48):17296-17301.

42. Hwang D, Smith JJ, Leslie DM, Weston AD, Rust AG, Ramsey S, de Atauri P, Siegel AF, Bolouri $\mathrm{H}$, Aitchison JD, et al: A data integration methodology for systems biology: experimental verification. Proceedings of the National Academy of Sciences of the United States of America 2005, 102(48):17302-17307.

doi:10.1186/1471-2164-15-S9-S5

Cite this article as: Hung et al:: The Yeast Nucleosome Atlas (YNA)

database: an integrative gene mining platform for studying chromatin structure and its regulation in yeast. BMC Genomics 2014 15(Suppl 9):S5.

\section{Submit your next manuscript to BioMed Central and take full advantage of:}

- Convenient online submission

- Thorough peer review

- No space constraints or color figure charges

- Immediate publication on acceptance

- Inclusion in PubMed, CAS, Scopus and Google Scholar

- Research which is freely available for redistribution 